\title{
Edge waves and resonance on elastic structures: an overview
}

\author{
by \\ Jane B. Lawrie and Julius D. Kaplunov \\ Department of Mathematics \\ Brunel University, Uxbridge, Middlesex, UB8 3PH. \\ jane.lawrie@brunel.ac.uk
}

\begin{abstract}
Over 50 years have elapsed since the first experimental observations of dynamic edge phenomena on elastic structures, yet the topic remains a diverse and vibrant source of research activity. This article provides a focused history and overview of such phenomena with particular emphasis on structures such as strips, rods, plates and shells. Within this context, some of the recent research highlights are discussed and this special issue ${ }^{1}$ of the journal Mathematics and Mechanics of Solids on dynamical edge phenomena is introduced.
\end{abstract}

\section{Introduction}

Edge resonance is a phenomenon that has intrigued engineers and mathematicians for over 50 years; not least because for much of that time it has defied full explanation but also, more recently, due to its potential interference with non-destructive evaluation (NDE) methods. Indeed, complicated waveforms arise due to the interaction of Rayleigh-Lamb waves diffracted both by an edge and a defect close to the edge, and this makes it difficult to recognise and interpret those modes generated by the defect [1]. The related phenomenon of edge waves is also of intrinsic interest and, along with other forms of guided wave, has potential applications in the measurement of material properties and NDE of thin elastic structures such as aircraft wings, submarine hulls etc.

In view of the potential importance of edge phenomena in NDE, it is worthwhile briefly discussing some of the pertinent developments in this field. NDE is a rich and well developed industry with many and diverse applications, for example, the detection of defects in pipelines [2]. A cylindrical guided wave can propagate considerable distances along a pipe wall and will be reflected at a discontinuity (in this context an area of corrosion). An overwhelming advantage of this inspection method is that there is no need to expose the pipeline by, for example, removing lagging or, in the case of underground pipes, extensive excavation. For this reason a significant body of research has explored the possibility of using the same principles for inspecting structures composed of two-dimensional, plate-like elements (which may also be large and not easily accessible). The difficulty here is that plate waves can propagate in any direction from a source and the associated energy decays with distance. Further, structures composed of thin elastic elements usually contain features such as welds, ribs or edges; themselves sources of scattering or resonance. Not only are such features potential sources of scattering, they also guide waves. Attention has thus

\footnotetext{
${ }^{1}$ The idea for this special issue was conceived following a specialist meeting of the CNRS funded "Groupement de Recherche 2501" Guided Waves Sub-group at Brunel University in March 2008.
} 
shifted towards the possibility of using "feature-guided" modes as a means of detecting cracks and defects close to welds or edges (a specific example is the formation of cracks at the edge of turbine or propeller blades). A correct understanding of the underlying physical and mathematical principles is, of course, essential for effective utilisation of these waves. The existence of "weld-guided" modes which propagate along a weld with little loss of energy has only recently been established [3]-[5]. On the other hand, although edge phenomena have been studied for over half a century significant new results still appear regularly in the literature, [6]-[8].

This article (indeed this special issue) is devoted to dynamic edge phenomena. The aims herein are to give a brief mathematical overview of the most prevalent edge phenomena occurring in elastic structures, demonstrate their basic physical properties and present an account of the major theoretical developments since their first discovery. A convenient starting point is the equations governing classical linear isotropic elasticity. Under the assumption of small displacement gradients and using a Cartesian co-ordinate system $\left(x_{1}, x_{2}, x_{3}\right)$, these may be expressed as

$$
\frac{\partial \sigma_{i j}}{\partial x_{j}}=\rho \frac{\partial^{2} u_{i}}{\partial t^{2}}
$$

where $\sigma_{i j}$ are the elements of the stress tensor, $\rho$ is the density of the elastic medium, $u_{i}$ is the displacement in the direction $x_{i}$ and $t$ denotes time. The usual summation convention applies to terms in which any subscript occurs twice. The stresses are expressed in terms of the displacements by

$$
\sigma_{i j}=\lambda e_{i i} \delta_{i j}+2 \mu e_{i j}
$$

where $\lambda$ and $\mu$ are the Lamé constants, $\delta_{i j}$ is the Kronecker delta function and

$$
e_{i j}=\frac{1}{2}\left(\frac{\partial u_{i}}{\partial x_{j}}+\frac{\partial u_{j}}{\partial x_{i}}\right)
$$

Various approximations to boundary values problems involving these equations are available. In particular, the cases of plane strain, plane stress and the related Kirchhoff and Kirchhoff-Love theories are considered.

The paper is structured as follows. Section 2 focuses on the phenomenon of edge resonance under plane strain. The major theoretical developments in this area since its first observation [11] in 1956 are summarised and discussed. The case of plane stress is considered in section 3. The existence of extensional edge waves is briefly explored and a simple example is presented which demonstrates how, under certain circumstances, these are related to edge resonance. The flexural Konenkov edge wave is the focus of Section 4. Recent work in this area is reviewed and, again, a simple example explores its relationship to edge resonance. The related topic of edge waves on shells is summarised in section 5. Section 6 draws upon the material discussed in sections 2-4 to present a coherent account of three dimensional edge waves. Both mixed and traction free face conditions are considered, the correspondence between the cut-on for the higher order edge modes and edge resonance is demonstrated and the current literature is reviewed. The concluding section highlights some areas of current research and, in this context, introduces the following five articles of this special issue. 


\section{Edge resonance under plane strain}

Edge resonance occurs for symmetric vibration at frequencies for which only the fundamental eigenmode can propagate ${ }^{2}$. It is characterized by the excitation of a localized motion on the plate end face (or edge). The phenomenon was first observed experimentally by Shaw [11] whilst studying the vibrations of a thick circular barium titanate disk. It was demonstrated that, for an isolated frequency below the lowest cut-on for the modes in an equivalent infinite layer, resonant vibration concentrated near the disc circumference. The first analytic verification of this phenomena used a plate theory to obtain an approximate value for the resonance frequency [12]. Subsequently, numerous theoretical studies attempted to explain such phenomena arising in elastic bodies of canonical shape.

The most commonly addressed geometrical configuration is an elastic slab under plane strain occupying the space, say, $x_{1}>0,-\infty<x_{2}<\infty-\bar{h} \leq x_{3} \leq \bar{h}$, and in which the edge at $x_{1}=0,-\bar{h} \leq x_{3} \leq \bar{h}$ is traction free: that is, $\sigma_{11}=\sigma_{13}=0$. It is worth recalling that plain strain is defined as that state in which the strain normal to the $x_{1} x_{3}$ plane, $e_{22}$, and the shear strains $e_{21}$ and $e_{23}$ are zero. Under this assumption the governing equation (1.1) reduces to the two-dimensional case with $u_{2}=0, \frac{\partial}{\partial x_{2}}=0$ and such configurations are thus commonly referred to as semi-infinite strips.

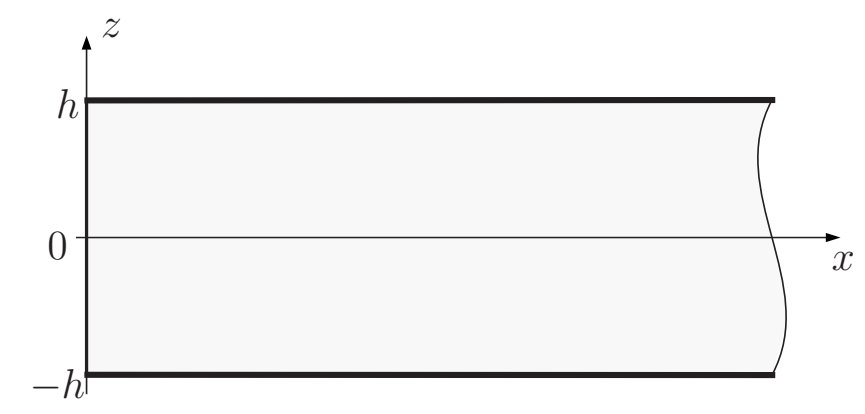

Figure 1: The configuration of the non-dimensional semi-infinite strip.

On assuming harmonic time dependence such that

$$
u_{1}\left(x_{1}, x_{3}, t\right)=\Re\left\{u\left(x_{1}, x_{3}\right) e^{-i \omega t}\right\}, \quad u_{3}\left(x_{1}, x_{3}, t\right)=\Re\left\{w\left(x_{1}, x_{3}\right) e^{-i \omega t}\right\}
$$

it is convenient to non-dimensionalise with respect to time and length scales $\omega^{-1}$ and $k^{-1}$ such that $k x_{1}=x, k x_{3}=z$ where $k=\omega / c_{p}$ and $c_{p}=(\lambda+2 \mu) / \rho$. Note that, the strip half-width thus becomes $h=k \bar{h}$ (see figure 1) and stresses such as $\sigma_{13}$ are henceforth referred to as $\sigma_{x z}$ etc. The governing equations may then be expressed as

$$
\tau^{2} \nabla^{2} \underline{u}+\left(1-\tau^{2}\right) \nabla(\nabla \cdot \underline{u})+\underline{u}=0 .
$$

Here $\tau$ is the ratio of the shear wave speed $c_{s}$ to that of the longitudinal waves $c_{p}$, thus:

$$
\tau^{2}=\frac{c_{s}^{2}}{c_{p}^{2}}=\frac{\mu}{(\lambda+2 \mu)}=\frac{1-2 \nu}{2(1-\nu)}
$$

\footnotetext{
${ }^{2}$ The term "fundamental" implies that the mode propagates even as the frequency tends to zero.
} 
where $\nu$ is Poisson's ratio. The non-dimensional time independent displacement vector is given by $\underline{u}=(u(x, z), w(x, z))$ and, for waves that are symmetric about $z=0$ and travel in the positive $x$ direction, separable solutions may be written as

$$
u(x, z)=U(z) e^{i \alpha x} \quad \text { and } \quad w(x, z)=W(z) e^{i \alpha x}
$$

where $U(-z)=U(z)$ and $W(-z)=-W(z)$. The form of the functions $U(z)$ and $W(z)$ and admissible values for the wavenumber $\alpha$ depend on the conditions applied along the faces $z= \pm h$. In the case of traction free faces the classical Rayeigh-Lamb (RL) modes are generated (see section 6) for which the wavenumbers are the roots of the characteristic equation:

$$
\left(\alpha^{2}+\delta^{2}\right)^{2} \cosh (\gamma h) \sinh (\delta h)-4 \alpha^{2} \delta \gamma \sinh (\gamma h) \cosh (\delta h)=0
$$

where $\gamma=\left(\alpha^{2}-1\right)^{1 / 2}$ and $\delta=\left(\alpha^{2}-1 / \tau^{2}\right)^{1 / 2}$. This is the case that has received the most attention in the literature to date.

A natural starting point for examining the phenomena of edge resonance is to express the displacement field as a superposition of the RL waves. Indeed, a range of methods based on such modal expansions have been employed. For example, Torvik [13] used a truncated modal expansion to obtain an approximation to the resonance frequency for a strip with $\nu=0.31$. It was found that

$$
\omega \approx 1.483 \frac{\pi c_{s}}{2 \bar{h}}
$$

a result that was subsequently also obtained using an approach involving both the RL modes and a variational formulation [14]. This approximation is similar to but more accurate than that obtained using plate theory [12]. Gregory and Gladwell [15] used RL modal expansions to investigate the distribution of energy amongst the various reflected modes generated by an incoming fundamental mode. In that study the edge resonance is manifest as very large amplitudes for eigenmodes with complex wave numbers. More recent publications combine modal, finite element and experimental approaches to study the edge mode vibration, for example [1].

It is remarkable that, despite the various attempts to predict the resonance frequency, for over 30 years it remained unclear whether edge resonance in a semi-infinite waveguide corresponds to a true trapped mode. Indeed, Gregory and Gladwell [15] specifically comment on this conundrum, although the numerical results of an earlier article [16] suggested that, at least in the case $\nu=0$, a trapped mode was likely. In 1998 a rigorous variational proof of the existence of a real eigenvalue relating to a true edge resonance in a semi-infinite elastic strip was presented by Roitberg et al [6] for $\nu=0$. This significant result was to provide a stepping stone for future work. The following points, whilst not providing a full explanation, prove to be crucial in terms of extending the work to non-zero values of $\nu$. In the special case $\nu=0$ and at frequencies below the first cut-on, the fundamental symmetric RL wave is orthogonal to the evanescent modes [16]. In this case, the resonant eigenvalue can be computed from an infinite set of algebraic equations expressing a linear dependence of the evanescent modes at the edge. Thus, the edge boundary conditions are satisfied with no loss of energy from the edge zone.

It was subsequently shown [7], [8] that a real eigenvalue also occurs at $\nu \approx 0.2248$ 
when the fundamental RL mode coincides with a Lamé mode ${ }^{3}$ and again is orthogonal to the evanescent modes. A true edge resonance does not appear for other values of Poisson's ratio, however, complex-valued resonance frequencies do occur. In these cases there is some loss of vibrational energy due to the radiation by the fundamental mode. Inspection of numerical data presented in the aforementioned publications indicates that these losses are relatively small. Thus, it is to be expected that a resonant-like behaviour will occur over the whole interval $0 \leq \nu \leq 0.5$. The approximate empirical formula for the real part of the resonance frequency proposed in [7] is:

$$
\Re(\omega) \approx \frac{c_{s}}{\bar{h}}\left(0.652 \nu^{2}+0.898 \nu+1.9866\right) .
$$

The equivalent formula given in [8] is almost identical with each coefficient agreeing with those above to two decimal places.

The above discussion has focused on the edge resonance observed on a semi-infinite strip under the assumption of plane strain and traction free faces at $z= \pm h$, and at frequencies below the first cut-on. Complex resonances may also occur at frequencies with real parts above the first cut-on; although the mechanisms by which these occur will necessarily be more complicated. Further, edge resonance occurs for strips with mixed or even fixed face conditions and in these cases the underlying analysis is very often more straight forward; a case of mixed face conditions is remarked upon in the footnote at the end of section 3 and the topic is discussed further in section 6 .

It is worth mentioning a number of analogous phenomena. Firstly, interfacial resonance is known to occur on the boundary between two joined half-strips with different elastic properties [17]. Secondly, as might be expected, edge resonance also occurs for a semi-infinite elastic circular cylindrical rod [18]-[23]. In this case, the Pochammer-Chree modes are the counterparts of the RL modes for a strip. Finally, a thorough analysis of the edge resonances in finite strips and cylinders is given in [24].

\section{$3 \quad$ Extensional edge phenomena on plates}

The plane stress plate model has traditionally been derived under the ad-hoc assumptions that: all loads act in and are symmetric about the mid-plane; the in-plane displacements, strains and stresses are uniform through the plate thickness and the normal and shear components of stress in the direction perpendicular to the plate are zero or negligible. For a semi-infinite plate with its mid-plane coinciding with the $x_{1} x_{2}$ plane, $x_{1}>0$, the latter point implies that $\sigma_{33}=\sigma_{31}=\sigma_{32} \approx 0$ (but $\left.u_{3} \neq 0\right)$ and this clearly reduces (1.1) from three to two equations. A more mathematical and generic point of view, however, is that the plane stress model is the leading order long wave, low frequency limit for extensional motion. Under these asymptotic assumptions it can be derived rigorously see; for example [10].

On assuming harmonic time dependence and non-dimensionalising in a manner analogous to that in section 2 , it is found that the plane stress governing equation is

\footnotetext{
${ }^{3}$ The Lamé modes are a special case of the Rayleigh-Lamb modes in which (2.7) is satisfied by simultaneously choosing $\delta h=i\left(\frac{\pi}{2}+n \pi\right)$ and $\left(2 \delta^{2}+1 / \tau^{2}\right)=0$. They thus exist only at discrete frequencies defined by $\omega \bar{h}=\sqrt{2} c_{s}\left(\frac{\pi}{2}+n \pi\right)$.
} 
formally identical to (2.4), however, the definition of $\tau$ differs. In this case

$$
\tau^{2}=\frac{c_{s}^{2}}{\bar{c}_{p}^{2}}=\frac{\lambda}{(\lambda+2 \mu)}=\frac{1-\nu}{2}
$$

where the wave speeds $\bar{c}_{p}$ and $c_{s}$ are defined by

$$
c_{s}=\sqrt{\frac{E}{2 \rho(1+\nu)}}, \quad \bar{c}_{p}=\sqrt{\frac{E}{\rho\left(1-\nu^{2}\right)}}
$$

in which $E$ is Young's modulus and, as above, $\nu$ is Poisson's ratio and $\rho$ is the density of the plate.

This plate configuration, shown in figure 2, permits perhaps the simplest example of an extensional edge wave, having the form:

$$
u(x, y)=e^{i \xi y}\left(e^{-\gamma x}+A e^{-\delta x}\right), \quad v(x, y)=-\frac{i e^{i \xi y}}{\gamma \xi}\left(\xi^{2} e^{-\gamma x}+\gamma \delta A e^{-\delta x}\right)
$$

where $\gamma=\left(\xi^{2}-1\right)^{1 / 2}$ and $\delta=\left(\xi^{2}-1 / \tau^{2}\right)^{1 / 2}$. The coefficient $A$ and wavenumber $\xi$ are determined using the traction free conditions: $\sigma_{x x}=\sigma_{x y}=0$ at $x=0$. It is found that $A=\left(\xi^{2} \nu-\gamma^{2}\right) /(\gamma \delta(1-\nu))$ and the extensional edge wavenumber, $\xi_{e}$, is the real root of $\Delta_{e}(\xi)=0$ where

$$
\Delta_{e}(\xi)=\left(\xi^{2}+\delta^{2}\right)^{2}-4 \xi^{2} \delta \gamma
$$

This wave is the analogue of the classical Rayleigh surface wave [25] in the theory of plane strain. Its phase speed is given by

$$
c_{e}^{2}=\frac{E}{\rho\left(1-\nu^{2}\right) \xi_{e}^{2}} .
$$

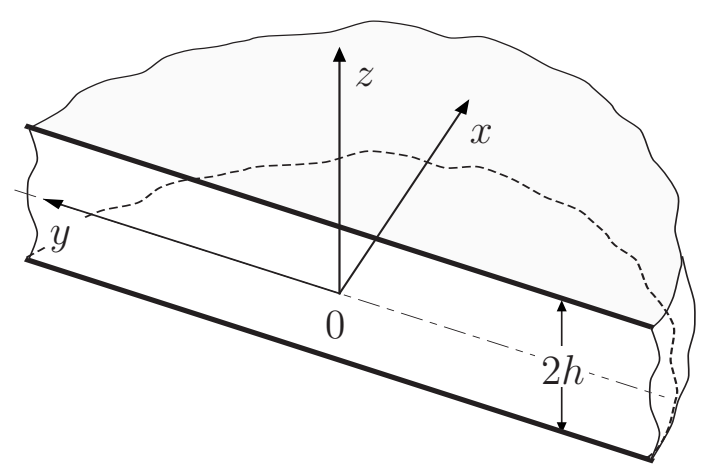

Figure 2: Configuration of the non-dimensional semi-infinite layer/plate.

In their experimental study, Oliver et al [26] associate the surface waves on the edge of a disc, with the plane stress model. The quasi-Rayleigh wave solution (3.11)(3.13), however, is valid in the low frequency limit, and so could only describe such waves at very low frequencies. Some effort was thus invested into developing plate theories to determine the behaviour of these waves for higher frequencies [27], [28], 
but it is now accepted that a fully three dimensional theory is then needed and this is discussed in section 6 .

The phenomenon of edge resonance discussed above in the context of plane strain, also occurs for semi-infinite strips (occupying, say, the region $x \geq 0,-a \leq y \leq a$ of the $x y$ plane where $a$ is related to the physical strip width by $a=k \bar{a}$ ) under plane stress. It is clear that, in addition to "traction free", other conditions are appropriate along the faces at $y= \pm a$. A simple example is the plane stress problem for a strip with a traction free edge at $x=0$ and the mixed boundary conditions $u=\frac{\partial v}{\partial y}=0$ along its semi-infinite horizontal edges. This gives rise to an infinite real valued edge spectrum associated with the quasi-Rayleigh standing edge mode. It is easily shown (see the appendix in [29]) that the displacements have the form

$$
u(x, y)=\sin \left(\frac{n \pi y}{a}\right)\left\{A_{n} e^{i \alpha_{n} x}+B_{n} e^{i \beta_{n} x}\right\}
$$

and

$$
v(x, y)=\cos \left(\frac{n \pi y}{a}\right)\left\{-\frac{i n \pi}{a \alpha_{n}} A_{n} e^{i \alpha_{n} x}+\frac{i a \beta_{n}}{n \pi} B_{n} e^{i \beta_{n} x}\right\}
$$

where $\alpha_{n}=\sqrt{1-n^{2} \pi^{2} / a^{2}}, \beta_{n}=\sqrt{1 / \tau^{2}-n^{2} \pi^{2} / a^{2}}$ and $n$ is an arbitrary integer. On applying the traction free conditions at $x=0$, it is found that

$$
B_{n}=\frac{-2 n^{2} \pi^{2} / a^{2}}{\left(n^{2} \pi^{2} / a^{2}-\beta_{n}^{2}\right)} A_{n}
$$

and either

$$
A_{n}=0 \quad \text { or } \quad \Delta_{e}(n \pi / a)=0 .
$$

It follows that edge resonance occurs when $n \pi=\xi_{e} a$, that is, at the discrete frequencies

$$
\omega_{n}=\frac{n \pi}{\bar{a}} c_{e}
$$

It is worth noting that, for any integer $n$, the resonance frequencies occur below the cut-on frequencies for the two travelling wave components of (3.14) and (3.15). Thus, the resonances are indeed trapped at the edge $x=0$.

In the above example the eigenfunctions $\cos (n \pi y / a), n=1,2,3, \ldots$ automatically satisfy the face conditions at $y= \pm a$. Thus, the traction free conditions at $x=0$ lead to the quasi-Rayleigh standing wave being generated for infinite spectrum of real frequencies. For other face conditions the situation is not so simple. The eigenfunctions have a more complicated structure and the traction free edge conditions can, in principle, be satisfied only if the plate displacement is expressed as a linear combination of all eigenfunctions and, furthermore, if the eigenfunctions exhibit some form of linear dependence. In particular, the edge resonance in a strip under plane stress in which all the edges are traction free cannot easily be related to the quasi-Rayleigh standing wave as in the above example. In this case, however, it is readily deduced ${ }^{4}$ [30] that the real eigenfrequencies appear for $\nu=0$ and $\nu \approx 0.29$. The latter value seems to be more relevant to engineering materials than its counterpart $\nu \approx 0.2248$ in the theory of plane strain.

\footnotetext{
${ }^{4}$ Note that on replacing $\nu$ in (3.10) with the quantity $\nu /(1-\nu),(2.5)$ is retrieved. This simple substitution enables the values $\nu=0$ and $\nu \approx 0.29$ to be calculated for edge resonance in plane stress. Likewise, the replacement of $\nu$ in $(3.18)$ with the quantity $\nu /(1-\nu)$ enables the edge resonance frequencies to be calculated for the case of plane strain and mixed face conditions.
} 


\section{Flexural edge phenomena on plates}

The leading order long wave, low frequency approximation to flexural plate waves can be deduced using Kirchhoff theory. The Kirchhoff plate equation is usually derived by balancing the shearing forces and the bending and twisting moments in the plate, see for example [9] and [31], but can be deduced from (1.1)-(1.3) using delicate asymptotic analysis [10]. On assuming harmonic time dependence, $e^{-i \omega t}$, the equation is conveniently non-dimensionalised with respect to time and length scales $\omega^{-1}$ and $k^{-1}$ where

$$
k=\left(\frac{2 \rho \bar{h} \omega^{2}}{D}\right)^{1 / 4} \quad \text { with } \quad D=\frac{2 E \bar{h}^{3}}{3\left(1-\nu^{2}\right)} .
$$

Here, as before, $E$ is Young's modulus, $\nu$ is Poisson's ratio and $\bar{h}$ is the plate halfthickness. The non-dimensional equation governing the flexural motion of a thin plate is

$$
\frac{\partial^{4} w}{\partial x^{4}}+2 \frac{\partial^{4} w}{\partial x^{2} \partial y^{2}}+\frac{\partial^{4} w}{\partial y^{4}}-w=0
$$

As in section 3 , it is assumed that the plate lies in the region $x>0,-\infty<y<\infty$ and that the edge along $x=0$ is traction free. In this context traction free implies that the bending moment $M_{x}$ and the effective Kirchhoff shear force, see [31], $V_{x}=Q_{x}+M_{x y, y}$ must both vanish at $x=0$. Thus,

$$
\frac{\partial^{2} w}{\partial x^{2}}+\nu \frac{\partial^{2} w}{\partial y^{2}}=0 \quad \text { and } \quad \frac{\partial^{3} w}{\partial x^{3}}+(2-\nu) \frac{\partial^{3} w}{\partial x \partial y^{2}}=0 \quad \text { on } \quad x=0 .
$$

It is easily shown that (4.20) supports waves of the form

$$
w(x, y)=e^{i \xi y}\left(e^{-\gamma_{1} x}+A e^{-\gamma_{2} x}\right)
$$

where $\gamma_{1}=\left(\xi^{2}-1\right)^{1 / 2}$ and $\gamma_{2}=\left(\xi^{2}+1\right)^{1 / 2}$ (see figure 2 for plate geometry but recollect that, for Kirchhoff theory, $h=k \bar{h}$ is small). On applying (4.21), the edge wavenumber, $\xi_{k}$, is found to be the real root of $\Delta_{k}(\xi)=0$ where

$$
\Delta_{k}(\xi)=\gamma_{1}\left(1+(1-\nu) \xi^{2}\right)^{2}-\gamma_{2}\left(1-(1-\nu) \xi^{2}\right)^{2} .
$$

It follows that $\xi_{k}=p^{1 / 2} /\left(p^{2}-4\right)^{1 / 4}$ where $p=2(1-\nu+\sqrt{1+2 \nu(\nu-1)}) / \nu^{2}$.

The discovery of this edge wave has an interesting history as reported by Norris et al [32]. The wave was first studied by Konenkov [33], however, due to the unfamiliarity of Soviet literature to many western researchers at that time, it was independently rediscovered some years later [35], [36]. What is less well known is that an article by Ishlinsky [34], preceding that by Konenkov, dealt with a near identical eigenvalue problem arising from the theory of plate stability. In contrast to the extensional wave predicted by the equations of plane stress, Konenkov's wave demonstrates dispersion and is sensitive to the plate thickness. Its phase speed is given by

$$
c_{k}^{2}=\frac{\omega \bar{h}}{\xi_{k}^{2}} \sqrt{\frac{E}{3 \rho\left(1-\nu^{2}\right)}} .
$$

The theory of flexural plate edge waves has been significantly developed in recent years, now taking into account anisotropy, layering, refined plate models [37]-[42] and 
the effects of fluid loading [43]. The existence and uniqueness of plate edge waves has been studied using the well-known Stroh formalism [44]-[46] and the Wiener-Hopf technique [47] has enabled the in depth study of edge waves generated on a semiinfinite crack in an otherwise infinite elastic plate [48]-[50]. Further, the multipole method has been used to investigate edge waves on a circular cut-out in a plate of finite width [51]. Flexural waves on an arbitrarily curved plate edge have also been studied [52].

As in the case of plane stress, edge resonance, also occurs for semi-infinite strips comprising Kirchhoff plates. The case of the dynamic bending of a Kirchhoff semiinfinite strip with a traction free vertical edge and simply supported horizontal faces ( $w=\frac{\partial^{2} w}{\partial y^{2}}=0$ on $\left.y= \pm a\right)$ is a simple example. It is easily shown [29] that the plate displacement can be expressed as:

$$
w(x, y)=\sin \left(\frac{n \pi y}{a}\right)\left(A_{n} e^{-i \alpha_{n} x}+B_{n} e^{-\beta_{n} x}\right)
$$

where $\alpha_{n}=\left(1-n^{2} \pi^{2} / a^{2}\right)^{1 / 2}$ and $\beta_{n}=\left(1+n^{2} \pi^{2} / a^{2}\right)^{1 / 2}, n=1,2,3, \ldots$ The coefficients $A_{n}$ and $B_{n}$ are chosen so that (4.21) are satisfied. On applying the first condition of (4.21), it is found that

$$
B_{n}=\frac{\left(\alpha_{n}^{2}+\nu n^{2} \pi^{2} / a^{2}\right)}{\left(\beta_{n}^{2}-\nu n^{2} \pi^{2} / a^{2}\right)} A_{n}
$$

whilst application of the second gives either

$$
A_{n}=0 \quad \text { or } \quad \Delta_{k}(n \pi / a)=0 .
$$

It follows that edge resonance occurs when $n \pi=\xi_{k} a$, that is

$$
\omega_{n}=\frac{n \pi}{\bar{a}} c_{k}
$$

As in the example for edge resonance on strip under plane stress, there is an infinite spectrum of real frequencies for which, in this case, the Konenkov standing wave can be generated at the edge. The resonance frequencies occur below the cut-on frequencies for the travelling wave component of (4.25), so the resonances are again trapped at the edge $x=0$.

\section{$5 \quad$ Edge waves on shells}

Localised waves also occur at the free edge of a thin semi-infinite circular cylindrical shell governed by Kirchhoff-Love theory [29]. The two plate edge waves discussed above coincide with the short-wave limit of the circumferential waves localised near the traction free edge. That is, as $n \rightarrow \infty$, formulae (3.18) and (4.28), in which $\bar{a} / \pi$ is the physical radius of the shell mid-surface (see figure 3 ), represent the leading order terms in the short wave asymptotic expansion of the extensional and bending edge eigenspectra. Although the shell curvature is small in this limit, it is not always negligible. The effect of shell curvature is apparent due the coupling between bending and extensional motions and leads to low level radiation damping of the extensional shell edge wave [29]. In addition, there exists a curvature borne super-low frequency 


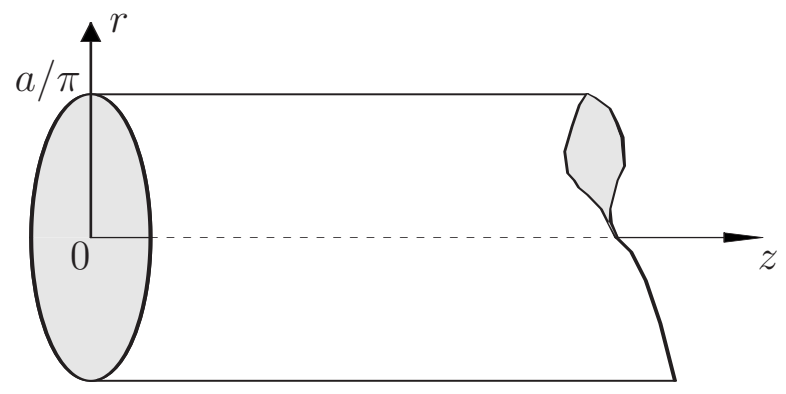

Figure 3: Non-dimensional configuration of the semi-infinite shell.

$\left(\omega \bar{a} / c_{e} \rightarrow 0\right)$ edge wave for which there is no analogue amongst plate edge waves. The latter are governed by "semi-membrane" shell theory [53].

Interestingly, free localised vibrations can also exist on shells for "partially clamped" edges (i.e. when motion in at least one direction is permitted). This is due to asymptotic separation of the boundary conditions for the extensional and bending components in the governing equations. For example, the curvature borne super-low frequency edge wave mentioned above also occurs for several variations of the "partially clamped" edge conditions. The important distinctions between shell and plate edge spectra arising due to shell curvature are fully discussed in [29].

More recent publications on the subject take into account the effect of anisotropy, more general shell shape and also develop a similar theory for interfacial shell waves (waves at the junction between two shell sections) [54]-[56]. It is also worth noting the similarity between localised edge phenomena and shell vibration and stability $[57]$.

\section{Three dimensional edge waves}

The edge waves occurring on a fully three dimensional plate in which the displacements are governed by (1.1)-(1.3) without approximation are more complicated than those considered hitherto. Naturally the two classes of edge wave considered in sections 3 and 4 are incorporated into the full three dimensional edge wave solutions as special cases. As in the two dimensional case, the structure of three dimensional edge waves, and indeed the complexity of the solution, is crucially influenced by the boundary conditions along the semi-infinite faces $z= \pm h, x>0$ (see figure 2).

Certain mixed boundary conditions on the plate faces permit simple sinusoidal variations of the displacement in the $z$ direction. For example, [58] for the face conditions $w=\sigma_{z x}=\sigma_{z y}=0$ on $z= \pm h$ it can be shown that the non-dimensional displacements that are symmetric about $z=0$ have the form:

$$
\begin{aligned}
u(x, y, z) & =e^{i \xi y}\left(e^{-\gamma x}+A e^{-\delta x}\right) \cos (n \pi z / h), \\
v(x, y, z) & =-\frac{i e^{i \xi y}}{\gamma \xi}\left(\xi^{2} e^{-\gamma x}+\gamma\left(A \delta-D \frac{n \pi}{h}\right) e^{-\delta x}\right) \cos (n \pi z / h), \\
w(x, y, z) & =e^{i \xi y}\left(\frac{n \pi}{h \gamma} e^{-\gamma x}+D e^{-\delta x}\right) \sin (n \pi z / h),
\end{aligned}
$$

where $\gamma=\left(\xi^{2}+\left(\frac{n \pi}{h}\right)^{2}-1\right)^{1 / 2}$ and $\delta=\left(\xi^{2}+\left(\frac{n \pi}{h}\right)^{2}-1 / \tau^{2}\right)^{1 / 2}$. This ansatz satisfies the face conditions and the governing equations. The coefficients $A, D$ and wavenumber 
$\xi(n)$ are determined using the traction free conditions: $\sigma_{x x}=\sigma_{x y}=\sigma_{x z}=0$ at $x=0$. It is found that the extensional edge wavenumber, $\xi_{3 d}(n)$, is the real root of $\Delta_{3 d}^{n}(\xi)=0$ where

$$
\Delta_{3 d}^{n}(\xi)=\left(\xi^{2}+\left(\frac{n \pi}{h}\right)^{2}+\delta^{2}\right)^{2}-4\left(\xi^{2}+\left(\frac{n \pi}{h}\right)^{2}\right) \delta \gamma .
$$

It is easily shown that, on recasting the independent variable as $\mathcal{K}=\sqrt{\xi^{2}(n)+\left(\frac{n \pi}{h}\right)^{2}}$, that (6.30) is the Rayleigh equation. Thus, the phase speed of this wave (taking both the $y$ and $z$ travelling wave components into consideration) is simply the Rayleigh speed, $c_{R}=c_{p} / \mathcal{K}$. The wave propagates, however, in the $y$ direction with speed $c_{3 d}(n)$ given by

$$
c_{3 d}^{2}(n)=\frac{E(1-\nu)}{\rho(1+\nu)(1-2 \nu) \xi_{3 d}^{2}(n)} .
$$

There are three important observations to be made. Firstly, the mode corresponding to $n=0$ is the fundamental mode and is the classical Rayleigh wave of plane strain. This mode has the same form as the extensional wave observed in the low frequency plane stress plate model (3.11), albeit with different definition of $\tau$. Secondly, it can be shown that $A \delta-D(n \pi / h)=-\xi^{2} \delta /\left(\delta^{2}+\mathcal{K}^{2}\right)$ and it follows that, when $\xi=0$, the displacement (6.29) is confined to the $x z$ plane reducing the geometry to a strip, as in figure 1. Then (6.30) takes exactly the same form as the resonance condition given in (3.17), except that $a$ is replaced by $h$ and, as mentioned above, the definition of $\tau$ differs. This clearly demonstrates the correspondence of the cut-on for each higher order three-dimensional edge mode with an edge resonance. Finally, the anti-symmetric modes for this set of mixed face conditions are obtained from (6.29) simply by replacing $n$ with $n+1 / 2$ and interchanging the sines and cosines. In this case, since the wavenumber is then given by $\xi_{3 d}=\sqrt{\mathcal{K}^{2}-(n+1 / 2)^{2} \pi^{2} / h^{2}}$, it is clear that there is no propagating mode for $\mathcal{K} h<\pi / 2$. Further, (6.30) in which $n$ is replaced by $n+1 / 2$ gives the resonance condition.

The eigenmodes for the mixed face conditions $u=v=\sigma_{z z}=0$ on $z= \pm h$ are similar in form to (6.29). The symmetric modes are obtained from (6.29) simply by replacing $n$ with $n+1 / 2$ and it is immediately clear that no symmetric mode propagates for $\mathcal{K} h<\pi / 2$. The anti-symmetric modes are obtained with equal ease by interchanging the sines and cosines in (6.29). Close inspection reveals that the $n=0$ mode is flexural in form but, except in the case $\delta=0$, fails to satisfy the traction free conditions at $x=0$ and must therefore be excluded from the set of edge eigenmodes. Resonance conditions for the symmetric modes is again given by (6.30) with $n$ replaced by $n+1 / 2$ and for the antisymmentric modes simply by (6.30).

For traction free faces the situation is more complicated [59], [60]. There are two families of symmetric travelling modes: the Rayleigh-Lamb (RL) modes

$$
\begin{aligned}
u^{R L}(x, y, z) & =\{\cosh (\gamma z)+A \cosh (\delta z)\} e^{i \xi y+i \alpha x}, \\
v^{R L}(x, y, z) & =\left\{\frac{\xi}{\alpha} \cosh (\gamma z)+B \cosh (\delta z)\right\} e^{i \xi y+i \alpha x}, \\
w^{R L}(x, y, z) & =\left\{-\frac{i \gamma}{\alpha} \sinh (\gamma z)-\frac{i}{\delta}(\alpha A+\xi B) \sinh (\delta z)\right\} e^{i \xi y+i \alpha x},
\end{aligned}
$$

and the shear modes for which $w^{S}(x, y, z)=0$ and

$$
u^{S}(x, y, z)=\cosh (\delta z) e^{i \xi y+i \alpha x}, \quad v^{S}(x, y, z)=-\frac{\alpha}{\xi} \cosh (\delta z) e^{i \xi y+i \alpha x} .
$$


In both the above $\gamma=\left(\xi^{2}+\alpha^{2}-1\right)^{1 / 2}$ and $\delta=\left(\xi^{2}+\alpha^{2}-1 / \tau^{2}\right)^{1 / 2}$ and the dispersion relations for these modes are respectively $(2.7)$ and

$$
\sinh (\delta h)=0 .
$$

With appropriate choice of $A$ and $B$, both the sets of eigenmodes satisfy the governing equations and the traction free face conditions on $z= \pm h$. They do not, however, satisfy the traction free conditions at $x=0$. For the case $\nu=0$ (for which $\tau^{2}=1 / 2$ ) the edge wave can be constructed [60] by expressing the displacement field as a linear combination of the first RL and the first shear modes. It is easily verified that the displacements then take the form (3.11) together with $w=0$, and that the traction free conditions on $x=0$ are satisfied only if $\xi$ is the real root of (3.12) with $\tau^{2}=1 / 2$. In general, however, there is no simple form for the edge wave. The problem of determining the edge wave is analogous to the problem of edge resonance under plane strain (section 2). The traction free edge conditions must be satisfied and, for fixed $\nu$, this can only be achieved for appropriate relationships between $\omega$ and $\xi$. The approach taken in [60] is to express the displacement field as a linear combination of both families of symmetric eigenmodes. The authors then present a variational proof of the existence of the three dimensional fundamental symmetric edge wave over the whole frequency range. Further, it is clarified that, as in the case of mixed face conditions, the first edge resonance of a semi-infinite strip with traction free faces coincides with the cut-on of the first higher-order symmetric three dimensional edge wave.

It is clear that the extensional quasi-Rayleigh wave of section 3 corresponds to the fundamental symmetric three dimensional edge wave in the low frequency limit. Further, in certain special cases, the fundamental mode corresponds exactly to the classical Rayleigh wave. Likewise, the fundamental three dimensional antisymmetric edge wave takes the form of a purely flexural wave which for traction free faces tends to Konenkov's wave in the low-frequency limit $h=k \bar{h} \rightarrow 0$. This was clearly demonstrated in a finite element and experimental study of the three dimensional antisymmetric flexural edge wave in which its phase velocity was compared with that of Konenov's wave [61]. Since then a number of authors have revisited this point, assessing the accuracy of various plate theories [32] and refined asymptotic models [41] by comparison with [61]. In a recent article [62] the three dimensional antisymmetric edge wave eigenmodes are studied using a approach which involves the superposition of two partial solutions. The problem is thus reduced to that of solving an infinite system of linear algebraic equations. Again the the phase velocity of the fundamental antisymmetric edge wave is confirmed by comparison with [61].

\section{Introduction to the special issue}

Sections 1-6 have presented a summary of the most prevalent forms of elastic edge phenomena in terms of their history and underlying physical properties. Of course, research continues due both to the intrinsic scientific and mathematical interest of such phenomena and also to their potential importance in industry especially in nondestructive evaluation. This special issue brings together some of the latest findings of both intrinsic and engineering interest. With regard to the former, the articles within this special issue present several new results which are briefly outlined below. 
With regard to the latter, two topics have particular significance. The first is the development of smart components. That is, components that not only form part of the integral structure but also allow continual monitoring for crack formation. The second is the development of sophisticated substances such as liquid-crystalline elastomers. As industry finds application for these non-standard materials, research is needed to address the subtle differences that are likely to occur in the presentation of edge phenomena in such media.

In the first article of this special issue Pagneux revisits the problem of edge resonance on a semi-infinite circular elastic cylinder [19]-[23]. The mathematical details are analogous to [7] enabling him to focus on the mechanics of the process without dwelling on the analysis. He demonstrates that, as in the case of the semi-infinite strip, a complex resonance exists for all physical values of $\nu$. A particular highlight of this paper is the presentation of a simple (linear) approximate formula equivalent to (2.9) for the real part of the resonance frequency.

Pichugin and Rogerson, in the second article, investigate the effect of pre-stress (finite static homogeneous pre-deformation) on the propagation of extensional edge waves using a generalisation of the classic plane stress theory. An explicit parameter analysis of the edge wave characteristic equation demonstrates that edge waves in prestressed plates are similar to the surface waves in pre-stressed media. A surprising and significant new discovery is the possibility of non-unique edge wave solutions.

With the development of smart components in mind, Abrahams and Lawrie employ the Wiener-Hopf technique [47] to analyse the effect of an electric current on the edge wave and diffracted field due to a semi-infinite crack in a non-ferrous Kirchhoff plate. The presence of the current introduces an additional term into the governing plate equation, [63], essentially altering the plate properties from isotropic to orthotropic. An interesting feature that thus arises is the dual dependence of the edge wave phase speed on frequency and current, resulting in two distinct asymptotic behaviours. The authors also present details of the bending/twisting moment intensity factors close to the crack tip.

The fourth article, by Fu and Kaplunov, promotes the use of the Stroh-Hamilton formulation [45]. The authors revisit the problem first considered in [29], see figure 3 , and recast the governing shell equations into the Stroh-Hamilton form. By this means the problem is reduced to a matrix Riccati equation together with an integral representation for the edge-impedance matrix. The vibration frequencies are efficiently calculated using an elegant root finding technique.

In the final article, Zakharov considers the effects of a thin nematic ${ }^{5}$ layer on wave propagation in some canonical structures. He first explores the surface waves on an elastic solid with a thin nematic coating. The dispersion curves for the quasiRayleigh and quasi-Love waves are generated and some interesting resonance effects are observed. Subsequently, he studies a Kirchhoff type plate with a thin nematic coating and demonstrates the existence of flexural edge waves.

To conclude, over 50 years have elapsed since the first observations of edge phenomena, yet the topic remains a diverse and vibrant source of research activity. This article has provided a focused history and overview of edge phenomena on elastic

\footnotetext{
${ }^{5} \mathrm{~A}$ nematic material is a rubber like liquid crystalline elastomer possessing strong anisotropy in both elastic and viscous properties, together with some additional freedom caused by long polymer chains and molecules. Such media can be analysed using an approximate linear low frequency model [64].
} 
structures with particular emphasis on strips, rods, plates and shells. Within this context, this special issue presents a range of new results demonstrating the current "state of the art".

\section{References}

[1] Le Clezio, E., Predoi, M.V., Castaings, M., Hosten, B. and Rousseau, M. Numerical predictions and experiments on the free-plate edge mode. Ultrasonics 41(1), 25-40 (2003).

[2] Alleyne, D. N., Pavlakovic, B., Lowe, M. J. S. and Cawley, P. Rapid, long range inspection of chemical plant pipework using guided waves. Insight 43, 93-101 (2001).

[3] Sargent, J. P. Corrosion detection in welds and heat affected zones using ultrasonic Lamb waves. Insight 48, 160-167 (2006 ).

[4] Postnova, J. and Craster, R.V. (2008) "Trapped modes in 3D topographically varying plates" IMA Jl Appl Math 73, 950-963.

[5] Fan, Z. and Lowe, M.J.S. Elastic waves guided by a welded joint in a plate. Proceedings of the Royal society of London A465, 2053-2068 (2009).

[6] Roitberg, I., Vassiliev, D., and Weidl, T. Edge resonance in an elastic semi-strip. Quarterly Journal of Mechanics and Applied Mathematics 51(1), 1-13 (1998).

[7] Pagneux, V. Revisiting the edge resonance for Lamb waves in a semiinfinite plate. Journal of the Acoustical Society of America, 120(2), 649-656 (2006).

[8] Zernov, V., Pichugin A.V. and Kaplunov, J. Eigenvalue of a semi-infinite elastic strip. Proceedings of the Royal Society of London, A462, 1255-1270 (2006).

[9] Timoshenko, S.P. and Woinoswsky-Krieger, S The theory of plates and shells. (second edition) McGraw-Hill International Editions, ISBN 0070858209, 1959.

[10] Kaplunov, J.D., Kossovich, L.Yu. and Nolde, E.V. Dynamics of Thin Walled Elastic Bodies. Academic Press, ISBN 978-0123975904, 1997.

[11] Shaw, E. A. G. On the resonant vibrations of thick barium titanate disks. Journal of the Acoustical Society of America, 28, 38-50 (1956).

[12] Gazis, D.G. and Mindlin, R.D. Extensional vibrations and waves in a circular disk and a semi-infinite plate. Journal of Applied Mechanics. 27, 541-547 (1960).

[13] Torvik, P. J. Reflection of wave trains in semi-infinite plate. Journal of the Acoustical Society of America. 41, 346-353 (1967).

[14] Auld, B.A. and Tsao, E.M. Variational analysis of edge resonance in a semi-infinite plate. IEEE Transactions on Sonics and Ultrasonics, 24, 317-326 (1977).

[15] Gregory, R.D. and Gladwell, I. The reflection of a symmetric Rayleigh-Lamb wave at the fixed or free edge of a plate. Journal of Elasticity, 13(2), 185-206 (1983).

[16] Grinchenko, V.T. and Meleshko, V.V. On the resonance for a semi-infinite strip. Prikladnaya Mekhanika 16, 58-63 (Engl Transl Sov Appl Mech) (1980). 
[17] Getman, I.P. and Lisitskii, O.N. Reflection and transmission of sound waves through the interfacial bounday of two joined elastic half strips. Journal of Applied Mathematics and Mechanics, 52, 1044-1048 (1998).

[18] Oliver, J. Elastic wave dispersion in a cylindrical rod by a wide-band short-duration pulse technique. Journal of the Acoustical Society of America, 29, 189-194 (1957).

[19] Zemanek, J. Experimental and theoretical investigation of elastic wave-propagation in a cylinder. Journal of the Acoustical Society of America, 51(1), 265-282 (1972) .

[20] Gregory, R.D. and Gladwell, I. Axisymmetric waves in a semi-infinite elastic rod. Quarterley Journal of Mechanics and Applied Mathematics, 42, 327-337 (1989).

[21] de Billy, M. End resonance in infinite immersed rods of different cross sections. Journal of the Acoustical Society of America, 100, 92-97 (1996).

[22] Holst, A. and Vassiliev, D. Edge resonance in an elastic semi-infinite cylinder. Applicable Analysis, 74, 479-495 (2000).

[23] Ratassepp, M., Klauson, A., Chati, F., Leon, F. and Maze, G., Edge resonance in semiinfinite thick pipe: Numerical predictions and measurements. Journal of the Acoustical Society of America, 124(2), 875-885 (2008).

[24] Grinchenko, V.T. and Meleshko, V.V. Harmonic oscillations and waves in elastic bodies. Kiev, Naukova Dumka, (in Russian) 1981.

[25] Rayleigh, J.W.S. On waves propagated along the plane surface of an elastic solid. Proceedings of the London Mathematical Society, S1-17(1), 411 (1885).

[26] Oliver, J., Press, F. and Ewing, M. Two-dimensional model seismology. Geophysics, 19(2), 202-219 (1954).

[27] McCoy, J.J. and Mindlin, R.D. Extensional waves along the edge of an elastic plate. Journal of Applied Mechanics, 30(1), 75-78 (1963).

[28] Sinclair, R. and Stephens, R.W.B. Velocity dispersion of waves propagating along the edge of a plate. Acustica, 24(3), 160-165 (1971).

[29] Kaplunov, J.D., Kossovich, L.Yu. and Wilde, M.V. Free localized vibrations of a semiinfinite cylindrical shell. Journal of the Acoustical Society of America, 107(3), 1383-1393 (2000).

[30] Kaplunov, J., Pichugin, A.V. and Zernov, V. Extensional edge modes in elastic plates and shells. Journal of the Acoustical Society of America, 125(2), 621-623 (2008).

[31] Graff, K. Wave motion in elastic solids. Dover Edition, Dover Publications Inc., New York 1991.

[32] Norris, A.N., Krylov,V.V. and Abrahams, I.D. Flexural edge waves and comments on A new bending wave solution for the classical plate equation[J.Acoust. Soc. Am.104,22202222(1998)]. Journal of the Acoustical Society of America, 107(3), 1781-1784 (2000).

[33] Konenkov, Y.K. A Rayleigh-type flexural wave. Soviet Physics Acoustics. 6, 122-123 (1960). 
[34] Ishlinskii, A.Y. On a limiting process in the theory of the stability of elastic rectangular plates. Dokl. Akad. Nauk SSSR 95(3), 477-479 (1954).

[35] Sinha, B.K. Some remarks on propagation characteristics of ridge guides for acoustic waves at low frequencies. Journal of the Acoustical Society of America, 56, 16-18 (1974).

[36] Thurston, R.N. and McKenna, J. Flexural acoustic waves along the edge of a plate. IEEE Transactions on Sonics and Ultrasonics, 21, 296-297 (1974).

[37] Norris, A.N. Flexural edge waves. Journal of Sound and Vibration. 171, 571-573 (1994).

[38] Zakharov, D.D. Konenkov's waves in anisotropic layered plates. Acoustical Physics, 48, 171-175 (2002).

[39] Thompson, I., Abrahams, I.D. and Norris, A.N. On the existence of flexural edge waves on this orthotropic plates. Journal of the Acoustical Society of America, 112, 1756-1765 (2002).

[40] Zakharov, D.D. and Becker, W. Rayleigh type bending waves in anisotropic media. Journal of Sound and Vibration, 261, 805-818 (2003).

[41] Zakharov, D.D. Analysis of the acoustical edge flexural mode in a plate using refined asymptotics. Journal of the Acoustical Society of America, 116(2), 872-878 (2004).

[42] Piliposian, G.T., Belubekyan,M.V. and Ghazaryan, K.B. Localized bending waves in a transversely isotropic plate. Journal of Sound and Vibration, 329, 3596-3605 (2010).

[43] Norris, A.N. and Abrahams,I.D. On the existence of flexural edge waves on submerged elastic plates. Proceedings of the Royal Society A456, 1559-1582 (2000).

[44] $\mathrm{Fu}, \mathrm{Y}$. Existence and uniqueness of edge waves in a generally anisotropic elastic plate. Quarterly Journal of Mechanics and Applied Mathematics. 56, 605-616 (2003).

[45] Fu, Y.B. and Brookes, D.W. Edge waves in asymmetrically laminated plates. Journal of Mechanics and Physics of Solids, 54, 1-21 (2006).

[46] Lu, P., Chen, H.B., Lee, H.P., and Lu C. Further studies on edge waves in anisotropic elastic plates. International Journal of Solids and Structures 44, 2192-2208 (2007).

[47] Lawrie, J.B. and Abrahams, I.D. A brief historical perspective of the Wiener-Hopf technique. Journal of Engineering Mathematics, 59, 351-358 (2007).

[48] Norris, A.N. and Wang, Z. Bending wave diffraction from strips and cracks on thin plates. Quarterly Journal of Mechanics and Applied Mathematics, 47, 607-627 (1994).

[49] Thompson, I. and Abrahams, I.D. Diffraction of flexural waves by cracks in orthotropic this elastic plates. I Formal solution. Proceedings of the Royal Society of London, A461, 3413-3436 (2005).

[50] Thompson, I. \& Abrahams, I.D. Diffraction of flexural waves by cracks in orthotropic this elastic plates. II Far field analysis. Proceedings of the Royal Society of London, A463, 1615-1638 (2007).

[51] Porter, R. Trapped waves in thin elastic plates. Wave Motion, 45, 3-15 (2007). 
[52] Cherednichenko, K. An asymptotic expansion of the boundary-layer type for flexural waves along the curved edge of a Kirchoff-Love elastic plate. Journal of Mathematical Sciences, 142(6) (2007).

[53] Goldenveizer, A.L. Theory of elastic thin shells. Pergammon Press, Oxford 1961.

[54] Gulgazaryan, G.R., Gulgazaryan, L.G. and Saakyan, R.D. The vibrations of a thin elastic orthotropic circular cylindrical shell with free and hinged edges. Journal of Applied Mathematics and Mechanics, 72, 312-322 (2008).

[55] Kaplunov, J.D. and Wilde, M.V. Edge and interfacial vibrations in elastic shells of revolution. Zeitschrift fur Angewandte Mathematik und Physik 51, 530-549 (2000).

[56] Kaplunov, J.D. and Wilde, M.V. Free interfacial vibrations in cylindrical shells. Journal of the Acoustical Society of America, 111, 2692-2704 (2002).

[57] Andrianov, I.V. and Awrejcewicz, J. Edge-localized effects in buckling and vibrations of a shell with free in circumferential direction ends. Acta Mechanica 173, 41-47 (2004).

[58] Kaplunov, J., Prikazchikov, D.A. and Rogerson, G.A. On three dimensional edge waves in semi-infinite isotropic plates subject to mixed face boundary conditions. Journal of the Acoustical Society of America, 118(5), 2975-2983 (2005).

[59] Meleshko V.V., Propagation of surface wave along the edge of semi-infinite elastic layer. Dopovidi Akademii nauk UkrSSR, Series A(3), 3336 (in Russian) (1982).

[60] Zernov, V. and Kaplunov, J. Three dimensional edge-waves in plates. Proceedings of the Royal Society of London, A464, 301-318 (2008).

[61] Lagasse, P.E. and Oliner, A.A. Acoustic flexural mode on a ridge of semi-infinite height. Electronics Letters, 12(1), 11-13 (1976).

[62] Krushynska, A.A. Flexural edge waves in semi-infinite elastic plates. Journal of Sound and Vibration. doi:10.1016/j.jsv.2010.11.002 (2011).

[63] Ambur, D.R., Hasanyan, D., Librescu, L. and Qin, Z. Diffraction of harmonic flexural waves in a cracked elastic plate carrying electrical current. Proceedings of the Royal Society of London, A461, 3542-3560 (2005).

[64] Fradkin, L.J., Kamotski, I. V., Terentjev,E. M. and Zakharov, D.D. Low-frequency acoustic waves in nematic elastomers. Proceedings of the Royal Society of London, A459, 2627-2641 (2003). 